Xenotransplantation. 1994 August ; 1(1): 3-7. doi:10.1111/j.1399-3089.1994.tb00044.x.

\title{
Clinical xenotransplantation
}

Thomas E. Starzl, Noriko Murase, Andreas Tzakis, John J. Fung, Satoru Todo, Anthony J. Demetris, Rafael Manez, Ignazio R. Marino, and Luis Valdivia

Pittsburgh Transplant Institute, University of Pittsburgh Medical Center, Pittsburgh, PA, U.S.A

\section{Abstract}

Two baboon liver xenografts transplanted to patients with B virus hepatitis supported life for 70 and 26 days but did not function optimally despite minimum or no histopathologic findings of overt humoral or cellular rejection in serial biopsies. However, there was evidence of complement activation in both cases, which in retrospect was thought to explain the unsatisfactory outcome. Strategies to deal with this problem are discussed.

\section{Keywords}

baboon; liver; complement activation; B virus hepatitis

\section{Introduction}

In June 1992 and January 1993, we made attempts at baboon-to-human liver xenotransplantation, both of which failed after 70 and 26 days. The patients whose original disease was B hepatitis had no evidence of viral reinfection during their posttransplant survival, and there was little histopathologic evidence of conventional humoral or cellular rejection of these livers. The clinical courses have been published in detail elsewhere [1,2], including the infectious complications that were the immediate causes of death: ruptured mycotic intracerebral aneurysm caused by aspergillus in Case 1, and peritonitis, secondary to an anastomotic leak at the jejunojejunostomy of the Roux-y biliary reconstruction, in Case 2. However, the underlying reason for failure in both cases was suboptimal xenograft function, which has not been satisfactorily explained.

\section{Surgical procedures}

The techniques were adapted from hepatic allotransplantation and seemed satisfactory. The body weights of the baboon donors were only $40 \%$ at those of the recipients, necessitating the so-called piggyback operation that leaves the recipient vena cava intact (Fig. 1). The livers regenerated up to optimal volume for recipient size in both cases. Biopsies showed multitudes of proliferating hepatocytes and duct cells. There were almost no infiltrating immunocytes. The interesting possibility has been raised recently by the studies of Shiraishi et al [3] that the use of small-to-large liver allografts (and by inference xenografts) unwittingly introduces an augmented antigenicity factor. In such circumstances, the increased MHC Class II expression that is independently associated with liver regeneration [4] appeared to be responsible for much more severe cellular and humoral rejections than seen with size-matched liver transplantation in the same rat strains. Murase had independently made the same observations in our laboratory (Table 1).

Address reprint requests to Thomas E. Starzl, MD, PhD, Department of Surgery, 3601 Fifth Avenue, 5C Falk Clinic, University of Pittsburgh, Pittsburgh, PA. 15213, U.S.A. 


\section{Preoperative status}

The conventional lymphocytotoxic crossmatch of the recipient sera with their donor lymphocytes was positive initially but negative after dithiothreitol (DTT) treatment, meaning that the anti bodies were largely IgM. Postoperatively, even the conventional crossmatches became negative. Both patients had donors of their own blood type, A to A in Case 1, and B to B in Case 2. They were immune competent with in vitro testing, although Patient 1 had asymptomatic HIV infection, and had undergone posttraumatic splenectomy 3 years previously. This patient was half the age ( 35 years) of Patient 2 and far less frail. Patient 2 had developed deep hepatic coma and was on a ventilator.

\section{Postoperative courses}

The first patient awoke promptly from anesthesia, resumed diet and ambulation, and became jaundice-free for most of the 70 days of survival. However, his alkaline phosphatase was elevated from the second week onward, suggesting partial biliary obstruction. At autopsy after 70 days, the entire biliary tree was filled with inspissated bile, and most of the biliary ducts, which by this time had become bile lakes, were denuded of epithelium.

The second patient never woke from his pre-existing coma, and in contrast to the first one failed to become anicteric. Again, the allograft at autopsy was filled with inspissated bile. Having the same cholestatic problem twice without clear evidence of mechanical obstruction raised the possibility that the baboon liver produced a lithogenic bile in the human environment. Although this kind of metabolic incompatibility has not been absolutely ruled out, it does not seem likely in view of other physiologic evidence of hepatic dysfunction. Neither patient was able to maintain a postoperative serum albumin above $2 \mathrm{gm} \%$. Both patients developed renal failure.

\section{Pathologic observations}

The disparity between the paucity of the histopathologic abnormalities, and the discouraging functional deficiencies of these transplants suggested incomplete control of xenograft rejection. Nearly 30 years ago, six baboon kidney xenografts were transplanted to patients treated with azathioprine-prednisone immunosuppression. The organs functioned for 6 to 60 days [5]. At the end, the baboon kidney xenografts had fierce cellular rejection. However, the key finding was a presumably antibody-mediated (humoral) occlusive endothelialitis of the graft vessels that choked off much of the arterial supply. The consequent distal ischemia appeared to be responsible for patchy gangrene of the xenografts, interspersed between islands of still-functional parenchyma. Similar gross and histopathologic findings were reported more than 20 years later by Bailey after cardiac xenotransplantation under a cyclosporine-based immunosuppressive regimen (the Baby Fae case) [6].

In contrast, the two liver xenografts treated with a four-drug immunosuppressive cocktail (FK 506, prednisone, prostaglandin $\mathrm{E}_{1}$, and cyclophosphamide) had little or no evidence of cellular rejection in biopsies obtained over the 70 and 26 days of survival. The patient with the longest follow-up had a mild focal cellular rejection on day 12 by the conventional criteria used for hepatic allografts. On day 64 there was a mild but diffuse increase in $\mathrm{T}$ (CD3+) and NK (Leu-7+) cells in the sinusoids and septal bile ducts but the findings were insufficient for an unequivocal diagnosis of rejection. There was some centrilobular hepatocyte drop out. No definite evidence of cellular rejection was seen in any of the seven biopsy samples taken from Patient 2 over a 26-day period. Both xenografts were entirely free of the arteritis that has been associated with vascular rejection in all previous baboon to human kidney or heart grafts. Nevertheless, sludging as well as the presence of 
polymorphonuclear leukocytes was seen in the sinusoids of the xenografts immediately after reperfusion, compatible with the diagnosis of an aborted hyperacute rejection.

Striking microchimerism of the kind associated with successful liver allotransplantation [7] was evident throughout life and at autopsy in both patients [1,2]. Because the first received only a liver, baboon DNA found by PCR in essentially all tissues retrieved at autopsy at 70 days were apparently chimeric nonparenchymal cells (NPCs). In addition to the liver, Patient 2 was given a large dose of unpurged bone marrow cells $\left(3 \times 10^{8} / \mathrm{kg}\right.$ body weight $)$ perioperatively. He also had mixed xenogeneic chimerism at all times until death.

\section{The question of complement activation Injury}

In both patients, total complement was depleted for most of the critical first 2 weeks while complement components $\mathrm{C}_{3}, \mathrm{C}_{4}$, and $\mathrm{C}_{5}$ became undetectable [1]. During this time, circulating immune complexes appeared. This complement evolution was similar to that reported previously in recipients of allografts transplanted across a positive lymphocytotoxic crossmatch [8]. After 10 days, the complement system settled down but irreversible damage may have occurred. Although the xenografts had little evidence of cellular or vascular rejection, they exhibited a very fine microsteatosis on their first biopsies that became obvious within a few days, particularly in Case $2[1,2]$. This finding has been reported in cases of allotransplantation with inexplicable primary hepatic nonfunction. Although these findings receded, the microsteatosis is suspected to represent a sublethal injury that precluded long term success in either case. There was prompt binding of IgM and IgG in the grafts. The IgM but not the IgG largely disappeared from the graft tissues in later biopsies.

The relationship of these findings to those of hyperacute allograft rejection has not been proved, but we believe that these livers were acutely damaged by an incomplete version of the kind of rejection first described in $\mathrm{ABO}$ incompatible kidney recipients [9] and subsequently in patients with a positive lymphocytotoxic crossmatch $(10,11]$. Although these syndromes usually are associated with anti-graft antibodies, this is not an absolute requirement $[12,13]$. The antibody versus nonantibody distinction in cases of hyperacute rejection described in the first instance the classical pathway of complement activation in which the first steps are antibody-dependent, and in the second, the alternative pathway, which does not require an antibody trigger or the participation of complement components $\mathrm{C}_{1}, \mathrm{C}_{2}$ and $\mathrm{C}_{4}$. The consequent hyperacute allograft rejection syndromes with or without preformed antigraft antibodies are not thought by us to be fundamentally different from those seen after xenotransplantation of organs between genetically diverse species [1,2]. We postulate that our baboon xenografts suffered damage by similar but less obvious mechanistic events.

\section{Control of complement activation: Back to the laboratory}

\section{Anticomplement strategies}

The complement pathogenicity that derives from the cleavage products of $\mathrm{C}_{3}$ and $\mathrm{C}_{5}$ has been effectively mitigated (but not eliminated with cobra venom (which depletes $\mathrm{C}_{3}$ and $\mathrm{C}_{5}$ ), and soluble recombinant complement receptor Type I, which binds to the cleavage fragments of $C_{3}$, and $C_{5}$, preventing amplification through $C_{3} b$. However, binding to the anaphylatoxins $\mathrm{C}_{3}$ a and $\mathrm{C}_{5}$ a prevents another mechanism of complement injury-the activation of mast cells, polymorphonuclear leukocytes, and other sources of soluble inflammatory mediators such as platelet-activating factor $[14,15]$. Thus, cobra venom and the soluble complement receptor 1 impede by different mechanisms both the classical or alternative cascades. By shutting off both pathways, such agents can interdict or ameliorate 
temporarily the Arthus reaction. Shwartzman reaction, and neutrophil-mediated tissue injury.

Another interesting anticomplement drug is a sesquiterpene compound called K76, which is produced by a species of fungi imperfecti found in the soil of one of the Okinawa islands. This drug blocks the $\mathrm{C}_{5}$ step of complement activation and also accelerates the decay of $\mathrm{C}_{5} \mathrm{~b}$, just proximal to the formation of the membrane attack complex (MAC) formed by complement components 5-9 in both the classical and alternative pathways of complement activation [16]. K76 has been used with another drug called FUT (a synthetic inhibitor of serine proteases) that had a small therapeutic effect by itself. K76 and FUT together allowed guinea pig heart graft survival in rats for $100 \mathrm{~min}$ [16].

We obtained a small supply of K76. which, when tested by Dr. Noriko Murase, gave superior results to those reported by Miyagawa et al. [16]; Miyagawa et al. had administered the drug intraperitoneally rather than intravenously. Median survival of guinea pig hearts in rats was increased by a single dose of $200 \mathrm{mg} / \mathrm{kg}$ from $8 \mathrm{~min}$ to more than $8 \mathrm{hr}$, and in one experiment to more than a day [2]. The surprising potency of intravenous K76 by itself or in combination with the other drug FUT has also been demonstrated in the very difficult pigto-dog kidney xenograft model. Survival out to $7 \mathrm{hr}$ was obtained, during which $200 \mathrm{mg} / \mathrm{hr}$ of urine was produced. However, in both the rodent and large animal xenograft models, the effect was limited to a few hours.

Brief control of the complement pathogenicity with any of the drugs or agents now available is not significant clinically. However, these drugs could be an adjunct to other strategies. One ancillary strategy could be the production of transgenic animals whose organs contain transfected human genes that regulate complement activation. This was a subject of intense interest at the recent international xenotransplant meeting in Cambridge.

\section{Complement change after experimental liver transplantation}

The need to prevent complement activation may be short-term, particularly if the transplantation is of the liver, which is the principal source of the body's complement and the sole source of most complement components such as $\mathrm{C}_{3}$. A paper by Valdivia at the Cambridge meeting has potential importance because it has demonstrated the species restriction of complement [17].

In Valdivia's investigation, the combination of hamster liver and its complement plus the rat recipient equaled a rat transferred to a hamster complement environment (Fig. 2). Valdivia showed how, in the new complement environment, hyperacute rejection of stable liver and heart xenografts could be precipitated with a small dose intravenous anti-hamster rat serum (Fig. 3). This was demonstrated to be a specific effect of the rat complement rather than the rat anti-hamster antibody because the hyperacute rejection could be completely avoided by simply heating the injected serum at $56^{\circ} \mathrm{C}$ for $30 \mathrm{~min}$ (Fig. 3). This allowed retention of the rat anti-hamster antibodies but removed the rat complement. This species restriction of complement is a discovery that may be exploitable to facilitate xenograft acceptance.

\section{Conclusions}

The xenograft barrier may be more vulnerable than most people realize at present. If complement activation proves to be the explanation, breaking this barrier whether it involves the classical or alternative pathway may not be unrealistic. Once this is achieved. it seems clear that conventional cellular and vascular rejection can be controlled with modern drug combinations. The complement reactions are the same as those that can abruptly destroy allografts. At present, the most intriguing question is whether the production of transgenic 
animals whose organs have complement regulatory proteins (such as DAF and CD59) will be helpful.

\section{Acknowledgments}

This work was aided by Project Grant No. DK29961 from the National Institutes of Health, Bethesda, Maryland.

\section{References}

1. Starzl TE, Fung J, Tzakis A, et al. Baboon to human liver transplantation. Lancet 1993;341:65. [PubMed: 8093402]

2. Starzl TE, Tzakis A, Fung JJ, et al. Prospects of clinical xenotransplantation. Transplantation Proc 1994;26:1082-1086.

3. Shiraishi M, Csete ME, Yasunaga C, et al. Regeneration-induced accelerated rejection reduced-size liver grafts. Transplantation 1994;57:336. [PubMed: 7509085]

4. Jonjic S, Radosevic-Stasic B, Cuk M, et al. Class II antigen induction in the regenerating liver of rats after partial hepatectomy. Transplantation 1987;44:165. [PubMed: 3474815]

5. Starzl TE, Marchioro TL, Peters GN, et al. Renal heterotransplantation from baboon to man: Experience with 6 cases. Transplantation 1964;2:752. [PubMed: 14224657]

6. Bailey L, Nehlsen-Cannarella S, Concepcion W, Jolley W. Baboon to human cardiac xenotransplantation in a neonate. JAMA 1985;254:3321. [PubMed: 2933538]

7. Starzl TE, Demetris AJ, Trucco M, et al. Cell migration and chimerism after whole organ transplantation: the basis of graft acceptance. Hepatology 1993;17:1127. [PubMed: 8514264]

8. Manez R, Kobayashi M, Takaya S, et al. Humoral rejection associated with antidonor lymphocytotoxic antibodies following liver transplantation. Transplant Proc 1993;25:888. [PubMed: 8442258]

9. Starzl TE, Marchioro TL, Holmes JH, et al. Renal homografts in patients with major donor-recipient blood group incompatibilities. Surgery 1964;55:195. [PubMed: 14121762]

10. Terasaki, PI.; Marchioro, TL.; Starzl, TE. Histocompatibility Testing. Washington. DC: National Acad Sci-National Res Council; 1965. Sero-typing of human lymphocyte antigens: Preliminary trials on long-term kidney homograft Survivors; p. 83-96.

11. Kissmeyer-Neilsen F, Olsen S, et al. Hyperacute rejection of kidney allografts, associated with preexisting humoral antibodies against donor cells. Lancet 1966;II:662.

12. Starzl TE, Lerner RA, Dixon FJ, et al. Shwartzman reaction after human renal transplantation. N Engl J Med 1968;278:642. [PubMed: 4866352]

13. Starzl TE, Boehmig HJ, Amemiya H, et al. Clotting changes, including disseminated intravascular coagulation, during rapid renal-homograft rejection. N Engl J Med 1970;283:383. [PubMed: 4193841]

14. Yeh CG, Marsh HC, Carson GR, et al. Recombinant soluble human complement receptor type I inhibits inflammation in the reversed passive arthus reaction in rats. J Immunol 1991;146:250. [PubMed: 1824590]

15. Pruitt SK, Bollinger RR. The effect of soluble complement receptor Type I on hyperacute allograft rejection. J Surg Res 1991;50:350. [PubMed: 1826935]

16. Miyagawa S, Shirakura R, Matsumiya G, et al. Prolonging discordant xenograft survival with anticomplement reagents K76COOH and FUT175. Transplantation 1993;55:709. [PubMed: 8475539]

17. Valdivia LA, Fung JJ, Demetris AJ, et al. Donor species complement after liver xenotransplantation: Mechanism of protection from hyperacute rejection. Transplantation 1994;57:918-922. [PubMed: 8154040] 


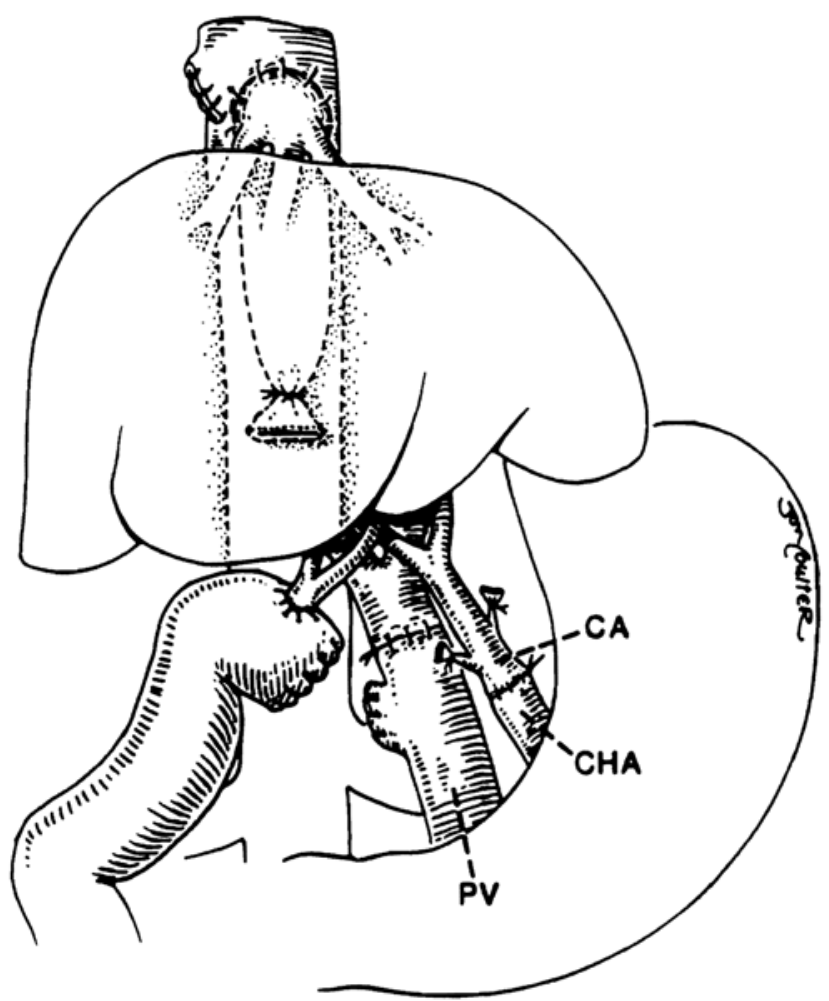

Fig. 1.

Piggyback operation used for both baboon-to-human xenotransplantations. 


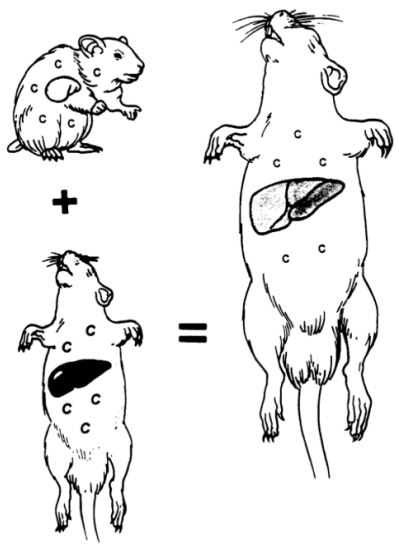

Fig. 2.

Demonstration by Valdivia et al. [17] that the complement environment of a xenograft recipient becomes that of the donor species. In these experiments, the dominant complement of rat recipients of hamster livers become hamster-specific within a few days, a change that was permanent. See text for further explanation. 


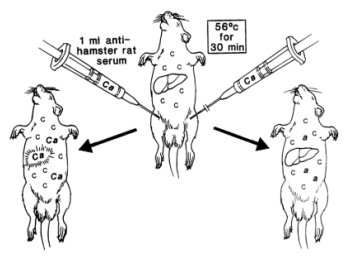

Fig. 3.

Second stage experiments of Valdivia et al. [17] following the engraftment shown in Figure 2 , showing species restriction of complement activation. Rats bearing long surviving hamster livers hyperacutely rejected these xenografts when injected with rat anti-hamster serum. but not if the serum was first decompleted by heating. 
Table 1

The effect of 70\% donor hepatectomy 1 day before liver transplantation ${ }^{a}$

\begin{tabular}{llrlr}
\hline Donor $\rightarrow$ Recipient & Donor hepatectomy (-24 hours) & $\boldsymbol{n}$ & Survival (days) & Median (days) \\
\hline LEW $\rightarrow$ BN & No & 10 & $23,23,23,25,28,29,29,30,32,37$ & 28.5 \\
& Yes & 5 & $7,8,9,11,12$ & 9.0 \\
\hline
\end{tabular}
$a_{\text {The recipient animals received no Immunosuppression. The role of rejection was established by histopathologic studies, and by control }}$
experiments showing that the hepatectomy effect could be abolished by a single dose of FK $506(1 \mathrm{mg} / \mathrm{kg} \mathrm{IM})$ on the day of transplantation. 